9th A. Friedmann International Seminar and

3rd Casimir Symposium 2015

International Journal of Modern Physics: Conference Series

Vol. 41 (2016) 1660135 (7 pages)

(C) The Author(s)

DOI: $10.1142 / \mathrm{S} 2010194516601356$

\title{
Quantum origin of suppression for vacuum fluctuations
}

\author{
Ja. V. Balitsky \\ Moscow Institute of Physics and Technology (State University), \\ Institutsky 9, Dolgoprudny, Moscow Region, 141701, Russia \\ balytskiyyaroslav@gmail.com \\ V. V. Kiselev \\ Russian State Research Center Institute for High Energy Physics \\ (National Research Centre Kurchatov Institute), Moscow Region, 141281, Russia \\ Moscow Institute of Physics and Technology (State University), \\ Institutsky 9, Dolgoprudny, Moscow Region, 141701, Russia \\ Valery.Kiselev@ihep.ru
}

Received 10 October 2015

Published 18 March 2016

\begin{abstract}
By example of a model with a spatially global scalar field, we show that the energy density of zero-point modes is exponentially suppressed by an average number of field quanta in a finite volume with respect to the energy density in the stationary state of minimal energy. We describe cosmological implications of mechanism.
\end{abstract}

Keywords: Cosmological constant problem; vacuum energy; scalar field.

PACS numbers: 03.70.+k, 98.80.Cq, 04.50.Kd

\section{Introduction and Recapitulation of the Cosmological Constant Problem}

After primary speculations presented in Ref. 1 we find that the cosmological constant ${ }^{2}$ is associated with a vacuum energy density generated by zero-point modes of quantum fields. ${ }^{3,4}$ In the framework of quantum field theory, these quantum fluctuations are ordinarily divergent and they should be renormalized. In this respect, the relevant renormalization is related to actual thresholds of energies, at which particles and forces contribute significantly. Then, one usually supposes that some combinations of Planckian scale and particle masses generate the energy density of vacuum $\rho_{v a c}$, so that the maximal estimate corresponds to the greater scale known in realsented physics and it yields $\rho_{v a c} \sim m_{P l}^{4}$, where the reduced Planck mass $m_{P l} \approx 2.4 \cdot 10^{18} \mathrm{GeV}$ is given by the Newton constant $\mathrm{G}$ as $8 \pi G m_{P l}^{2}=1$. Such types

This is an Open Access article published by World Scientific Publishing Company. It is distributed under the terms of the Creative Commons Attribution 4.0 (CC-BY) License. Further distribution of this work is permitted, provided the original work is properly cited. 
of estimates are in direct conflict with the value extracted from the cosmological data $^{5}$ giving $\rho_{v a c} \mapsto \rho_{\Lambda}=\Lambda^{4} \quad \Lambda \sim 10^{-3} \mathrm{eV}$, which is 30 orders of magnitude less than the Planck mass.

However, nobody can guarantee that the Planck scale defining the strength of the gravitational interaction has to establish a fundamental mass scale or energy threshold relevant to the cosmological constant. In this respect, one could follow a more realistic way by using the generally accepted description of particle physics in the Standard Model. So the direct observation of the Higgs boson allows us to evaluate the energy density of the electroweak vacuum from the effective potential of the Higgs boson in terms of the masses of the Higgs boson and W boson, which give $\rho_{\Lambda}^{E W} \sim m_{H}^{2} m_{W}^{2} \sim\left(10^{2} \mathrm{GeV}\right)^{4}$, exceeding the contribution due to the additional condensates in the quantum chromodynamics by at least 8 orders of magnitude. Then, the magnitude of mismatching the scale of the cosmological constant would be significantly relaxed from 30 orders to 14 orders, which is still significant. Notice that in this approach to the estimate of the cosmological constant scale, one ignores an arbitrary constant shift of the Higgs potential. This shift can originate from physics of other fields. In addition, at the observed mass value the Higgs potential can lose its stability at very large fields below the Planckian range due to effects of the renormalization group, which could produce the tunnel decay of the present Universe to another universe with a different vacuum.

Let us show that such a suppression can be explained due to a finite volume effect for quantum fluctuations in an excited nonstationary state. For the sake of clarity we exhibit the mechanism by considering a time-dependent scalar field $\phi(t)$. which is spatially global and free. The action in a finite physical volume is given by the expression

$$
S=V_{R} \int d t \frac{1}{2}\left(\dot{\phi}^{2}-m^{2} \phi^{2}\right),
$$

$\dot{\phi}=\frac{d \phi}{d t}$ and $m$ is the field mass. The specific reference frame of space-time suggestively should be associated with the reference frame of the homogeneous component of cosmic microwave background radiation in the Universe, so that time $t$ could correspond to the cosmic time of Friedmann-Robertson-Walker-Lemaitre metrics. The meaning of $V_{R}$ is the volume wherein the fluctuations of the field are causal, so that it can be considered as spatially global, while an inhomogeneity is evaluated by $|\nabla \phi| \sim \frac{\delta \phi}{\lambda_{C}}$, where $\lambda_{C}$ is the Compton length, $\lambda_{C}=\frac{1}{m}$ and $\delta \phi$ denotes the field fluctuation. The basic motivation and consideration are further considered in the field model of (1), so we ignore the influence of curved space-time on the main result for the moment. Formally, action (1) corresponds to the harmonic oscillator of frequency $\mathrm{m}$ and inertial mass $V_{R}$. Then, the dimensionless operators

$$
\begin{aligned}
\hat{Q} & =\frac{\phi}{\phi_{0}}, \quad \hat{P}=\frac{\dot{\phi}}{\dot{\phi}_{0}} \\
\phi_{0}^{2} & =\frac{1}{V_{R} m}, \quad \phi_{0}^{2}=\frac{m}{V_{R}}
\end{aligned}
$$


define the operators of annihilation and creation for the spatially global field quanta

$$
\hat{a}=\frac{1}{\sqrt{2}}(\hat{Q}+i \hat{P}), \quad \hat{a}^{+}=\frac{1}{\sqrt{2}}(\hat{Q}-i \hat{P}),
$$

with the standard commutator $\left[\hat{a}, \hat{a}^{+}\right]=1$. The Hamiltonian takes the form

$$
\hat{H}=\frac{1}{2} V_{R}\left(\dot{\phi}^{2}+m^{2} \phi^{2}\right)=\frac{1}{2}\left(\hat{P}^{2}+\hat{Q}^{2}\right)=m\left(\hat{a} \hat{a}^{+}+\frac{1}{2}\right) .
$$

If the field is nonstationary excited, its quantum state can be considered as a superposition of oscillatory coherent states, which minimize uncertainties in the field $\phi$ and its rate $\dot{\phi}$. Let us consider the coherent state $|\alpha\rangle$ with the average number of quanta $n$ in the volume $V_{R}$ :

$$
\hat{a}|\alpha\rangle=\alpha|\alpha\rangle, \quad \alpha^{*} \alpha=n, \quad \alpha=\frac{1}{\sqrt{2}}\left(Q_{0}+i P_{0}\right) .
$$

The averaged energy reads as $\langle E\rangle=\langle\alpha|\hat{H}| \alpha\rangle=m\left(n+\frac{1}{2}\right)$. Usually, the minimal energy shift of this state from the minimum of the potential is referred to as the energy level of zero-point mode (ZPM),

$$
\delta_{\min } E=\frac{1}{2} m
$$

So, it defines the quantity that we call the bare cosmological constant,

$$
\rho_{\Lambda}^{\text {bare }}=\frac{m}{2 V_{R}}=\langle v a c|\rho| v a c\rangle=m^{2}\left\langle v a c\left|\phi^{2}\right| v a c\right\rangle .
$$

This shows that the finite volume sets nonzero fluctuations of the spatially global field. If the fluctuations of the field match the Planckian scale, $\left\langle v a c\left|\phi^{2}\right| v a c\right\rangle \sim m_{P l}^{2}$, then the bare cosmological constant $\rho_{\Lambda}^{\text {bare }}$ takes a huge value, which constitutes the cosmological constant problem. On the other hand, if we restrict ourselves to the range of the Standard Model, then we expect that the mass of the scalar field is given by the mass of the Higgs boson $M$. So one can arrive at an estimate that is consistent with the expectations of the Standard Model, but it still would have a huge value.

However, in the next section we show that if the number of quanta for $\phi(t)$ is not equal to zero, $n \neq 0$, then only a fraction of an energy shift from the potential minimum refers to the ZPM and the fractional part of the energy does originate from the suppressed vacuum fluctuations; hence, the suppressed fraction indeed corresponds to the vacuum energy.

\section{Suppression Mechanism in the Action}

Let us find the fraction of the ZPM in the energy shift from the minimum of the potential for the coherent state. So, decomposing the state into the sum of the vacuum $|v a c\rangle$ and the state $|q u a n t a\rangle$ with nonzero numbers of stationary field quanta,

$$
|\alpha\rangle=A_{v a c}|v a c\rangle+A_{q}|q u a n t a\rangle
$$


we evaluate the average density of energy,

$$
\langle\alpha|\rho| \alpha\rangle=\left|A_{\text {vac }}\right|^{2}\langle v a c|\rho| v a c\rangle+\left|A_{q}\right|^{2}\langle, \text { quanta }|\rho| q u a n t a\rangle
$$

where the probability to find $k$ quanta in the coherent state is given by the Poisson distribution,

$$
\left|A_{k}\right|^{2}=\frac{n^{k}}{k !} e^{-n}
$$

while the free Hamiltonian does not mix the stationary states with different numbers of quanta.

Therefore, the average density of energy observed in gravity, is decomposed as

$$
\langle\rho\rangle=\left|A_{v a c}\right|^{2} \rho_{\Lambda}^{\text {bare }}+\rho_{q}
$$

at

$$
\left|A_{v a c}\right|^{2}=e^{-n}
$$

with $\rho_{q}$ being the energy density of nonzero-point modes that, for the coherent state, equals

$$
\rho_{q}=\frac{m}{V_{R}}\left(n+\frac{1}{2}-\frac{1}{2} e^{-n}\right)=\rho_{\Lambda}^{\text {bare }}\left(2 n+1-e^{-n}\right) .
$$

Thus, the true energy density generated by ZPMs in the coherent state is suppressed and is given by

$$
\rho_{\Lambda}=\left|A_{\text {vac }}\right|^{2} \rho_{\Lambda}^{\text {bare }} \mapsto e^{-n} \rho_{\Lambda}^{\text {bare }} .
$$

Relation (15) remains valid generically by the order of magnitude not only for the coherent state, but also for the most ordinary, nonexotic states yielding $\left|A_{v a c}\right|^{2} \sim$ $e^{-n}$.

We can hold the relation for the probability of the ZPM in the quantum state as the definition of the effective number of quanta in this state. Moreover, if the fluctuations of field quanta are statistically occasional, then the probability with respect to the number of quanta has to fit the Poisson distribution, and hence, the quantum state should be the coherent state.

The meaning of the bare cosmological constant is the following: if the system is in the vacuum state, the bare cosmological constant would be the energy density in the system, i.e., in the empty vacuum without any fields, particles, or quanta, only vacuum fluctuations. If the system is not empty and it is occasionally excited, the actual vacuum fluctuations are suppressed, which means the suppression of the observed cosmological constant. In this way, we assume in our model that there is not any different contribution to the cosmological constant, say, like some induced terms of various nature, since those additional terms cannot be suppressed in the same manner.

Let us look at the pressure of ZPMs in order to justify their vacuum status. The bare ZPMs have the $E^{\text {bare }}=\frac{1}{2} m$ independent of the reference volume. This means 
that the pressure is given by $p^{\text {bare }}=-\frac{\partial E}{\partial V}=0$, which can also be calculated by means of averaging the spatial components of the energy-momentum tensor,

$$
\left\langle v a c\left|T_{\beta}^{\alpha}\right| v a c\right\rangle=-\delta_{\beta}^{\alpha} p^{\text {bare }}=-\delta_{\beta}^{\alpha} \frac{1}{2}\left\langle v a c\left|\dot{\phi(t)^{2}}-m^{2} \phi(t)^{2}\right| v a c\right\rangle=0 .
$$

In contrast, the true energy of suppressed vacuum fluctuations in volume $V_{R}$ is

$$
E_{v a c}=V_{R} \rho_{\Lambda}=\frac{1}{2} m\left|A_{v a c}\right|^{2} .
$$

We can obtain the correct dependence on the volume if we make the vacuum density of energy constant, which implies $\frac{\left|A_{v a c}\right|^{2}}{V_{R}}=$ const and the pressure obtains the value,

$$
p=-\frac{\partial E}{\partial V_{R}}=-\rho_{\Lambda}
$$

hence,

$$
\frac{\partial n}{\partial \ln V_{R}}=-1
$$

Therefore, the reference volume exponentially declines with the growth of quantum number $n$, and there is a maximal number corresponding to a minimal volume of Planckian length. This derivation of the actual value for the parameter of the vacuum state is elementary, but it could be absolutely impossible if we ignored the variation of the suppression

Let us evaluate the relative inhomogeneity of the field with respect to the energy density. Thus, $\frac{|\nabla \phi|^{2}}{m^{2}\langle\phi\rangle} \sim \frac{m^{2}(\delta \phi)^{2}}{m^{2}\langle\phi\rangle} \sim \frac{\delta E}{E} \sim \frac{1}{\sqrt{n}} \ll 1$ Therefore, the inhomogeneity is negligible if the field is nonstationary excited to a large value of quanta.

\section{Cosmology and Model Estimates}

As we already emphasized, we ignored effects due to a curved space-time, while we derived our mechanism for the suppressed cosmological constant even though it is relevant to the system with gravity. In this respect, we assume that the relevant quantities can enter as the initial conditions for the further evolution of the system by taking into account the gravitational expansion. So the energy density of the vacuum remains constant, while the quanta and their energy densities follow the transformations in accordance with the relative field equations, taking into account the gravity as well.

Nevertheless, we have to mention that in the literature there are computations of energy density for the ZPMs in a curved background, which take into account the dependence on the space-time curvature. Modern investigations in Refs. 7 and 8 argue that in the curved space-time, for instance, in the de Sitter space-time close to the space-time of inflation in the early Universe the ZPMs themselves produce the energy density that quadratically evolves with the Hubble rate. We stress that 
such an effect means that the energy density of ZPM is not the cosmological constant at all, since the emergent equation of state (EOS) deviates from the vacuum equation of state, when the ratio of pressure to the energy density equals -1 and; hence, ZPMs generate the form of dark energy. In the form of expression (15), the described mechanism rigorously sets the quantum suppression of the bare cosmological constant. It is relevant to the cosmology because, at first, the spatially global scalar field could be associated with the spatially global part of the inflaton ; second, a finite volume of causal fluctuations corresponds to a primary volume of the Universe at the start of inflation, wherein the inflaton field can be considered as spatially global. In this way, we can make estimations in a simple manner, say, by setting the primary fluctuations of the field as

$$
\left\langle v a c\left|\phi^{2}\right| v a c\right\rangle \sim m_{P l}^{2} \Rightarrow \frac{1}{V_{R}} \sim m m_{P l}^{2}
$$

hence, the average number of the spatially global field quanta is evaluated by

$$
n=\ln \frac{\rho_{\Lambda}^{\text {bare }}}{\rho_{\Lambda}} \sim 275-2 \ln \frac{m_{P l}}{m} \gg 1,
$$

since the inflaton is quite heavy, i.e., $m \sim 10^{13}$ and $n \sim 250$. Since the mechanism should be accepted as real, we need to figure out what the number of $n$ is? Our studies show that the answer can be found, for instance, in the framework of the model with the inflaton nonminimally coupled to gravity, i.e., due to the interaction term of the Lagrangian in the form

$$
L_{i n t}=\frac{1}{2} \xi \phi m_{P l} R,
$$

where $R$ is the scalar curvature of the metric in the Jordan frame. In the Einstein frame, the inflation scale is $\Lambda_{\text {inf }} \sim 10^{16} \mathrm{GeV}$ while the transformed inflaton obtains the mass of the order of $\frac{\Lambda_{i n f}}{\xi}$. The parameters obey the relation for the strong coupling

$$
n \sim \xi \sim \frac{m_{P l}}{\Lambda_{\text {inf }}}
$$

We address this problem of the total cosmological constant in our new paper, ${ }^{6}$ wherein we discuss the pseudo-Goldstone nature of the inflaton with respect to the global shift of the energy scale of vacuum energy density. In this mechanism, the primary cosmological constant induced by all of the actual contributions is matched to the bare cosmological constant of inflaton. Our hope is that this matching solves the problem of copious ingredients of the total cosmological constant.

\section{Acknowledgments}

This work is supported by the Russian Foundation for Basic Research, Grant No. 14- 02-00096. 


\section{References}

1. Ya. Zeldovich, Sov. Phys. Usp. 11, 381 (1968).

2. S. Weinberg, Rev. Mod. Phys. 61, 1 (1989).

3. A. Andrianov, F. Cannata, P. Giacconi, A. Y. Kamenshchik and R. Soldati, Phys.Lett. B 651, 306 (2007).

4. E. K. Akhmedov, Vacuum energy and relativistic invariance, arXiv:hep-th/0204048.

5. P. Ade et al. (Planck), Astron. Astrophys. 571, A16 (2014).

6. Ja. V. Balitsky and V. V. Kiselev, Journal of Cosmology and Astroparticle Physics 10.1088/1475-7516/2015/04/032.

7. M. Maggiore, Phys. Rev. D 83, 063514 (2011).

8. L. Hollenstein, M. Jaccard, M. Maggiore and E. Mitsou, Phys. Rev. D 85, 124031 (2012). 